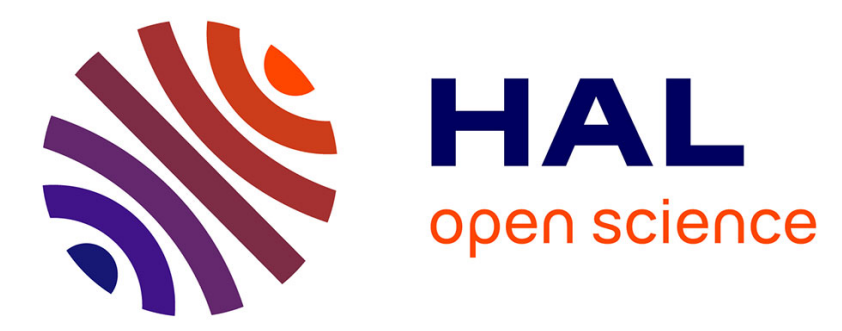

\title{
From single to multiple pulse all-optical switching in GdFeCo thin films
}

Yong Xu, Michel Hehn, Weisheng Zhao, Xiaoyang Lin, Grégory Malinowski, Stéphane Mangin

\section{- To cite this version:}

Yong Xu, Michel Hehn, Weisheng Zhao, Xiaoyang Lin, Grégory Malinowski, et al.. From single to multiple pulse all-optical switching in GdFeCo thin films. Physical Review B: Condensed Matter and Materials Physics (1998-2015), 2019, 100 (064424), 10.1103/PhysRevB.100.064424 . hal-02337730

\section{HAL Id: hal-02337730 \\ https://hal.univ-lorraine.fr/hal-02337730}

Submitted on 29 Oct 2019

HAL is a multi-disciplinary open access archive for the deposit and dissemination of scientific research documents, whether they are published or not. The documents may come from teaching and research institutions in France or abroad, or from public or private research centers.
L'archive ouverte pluridisciplinaire HAL, est destinée au dépôt et à la diffusion de documents scientifiques de niveau recherche, publiés ou non, émanant des établissements d'enseignement et de recherche français ou étrangers, des laboratoires publics ou privés. 


\title{
From single to multiple pulse all-optical switching in GdFeCo thin films
}

\author{
Yong Xu,${ }^{1,2}$ Michel Hehn, ${ }^{2}$ Weisheng Zhao, ${ }^{1, *}$ Xiaoyang Lin, ${ }^{1}$ Grégory Malinowski, ${ }^{2}$ and Stéphane Mangin $\circledast^{2, \dagger}$ \\ ${ }^{1}$ Fert Beijing Institute, BDBC, School of Microelectronics, Beihang University, Beijing 100191, China \\ ${ }^{2}$ Institut Jean Lamour, CNRS UMR 7198, Université de Lorraine, Nancy F-54000, France
}

(Received 20 September 2018; revised manuscript received 22 July 2019; published 26 August 2019)

\begin{abstract}
All-optical switching refers to the magnetization reversal induced by ultrashort laser pulses. Both single-pulse all-optical helicity-independent switching and multiple pulses all-optical helicity-dependent switching have been reported for GdFeCo thin films. In this paper, we demonstrate that for two GdFeCo compositions, the transition between these two behaviors can appear by increasing the thickness of the Pt capping or by tuning the laser fluence. The two types of switching cannot be observed for the same set of parameters, which supports that their mechanisms are uncorrelated. Our calculations indicate that the transition may be induced by a strong inhomogeneity of the electronic temperature within the thickness of the GdFeCo layer in the subpicosecond timescale.
\end{abstract}

DOI: 10.1103/PhysRevB.100.064424

\section{INTRODUCTION}

In the field of ultrafast magnetism, the subpicosecond demagnetization induced by femtosecond (fs) laser pulses was first observed in the pioneering experiment of Beaurepaire et al. [1]. Later, it was demonstrated that fs laser pulse could switch the magnetization without a magnetic field. This effect was called all-optical switching (AOS). AOS is considered a potential high-speed technique to write information in the field of magnetic data storage, memory, and logic devices [2].

In 2007, Stanciu et al. reported the deterministic magnetic switching, namely all-optical helicity-dependent switching (AO-HDS), in a GdFeCo thin film by sweeping a circularly polarized fs laser beam [3,4]. In 2014, Mangin et al. demonstrated that AO-HDS is a general effect of various perpendicularly magnetized materials, such as ferrimagnets, ferromagnets, and multilayers, etc. [5,6]. El Hadri et al. further demonstrated that AO-HDS requires multiple pulses to fully switch the magnetization [7].

On the other hand, Ostler et al. reported another type of AOS in GdFeCo alloys as well, namely all-optical helicityindependent switching (AO-HIS) $[8,9]$. This type of AOS is very different from the other. Indeed, a single laser pulse is enough to switch the magnetization. AO-HIS is only observed in the Gd-based samples: GdFeCo alloys, $\mathrm{Co} / \mathrm{Gd}$ multilayers [10], and spintronic devices [7,8,10-12]. Recent studies demonstrated a similar heat-driven single-pulse switching can be induced by a femtosecond electron-pulse or even a picosecond electrical pulse [13-15].

AO-HIS is often described using an atomic spin model. The ultrafast switching dynamics is triggered by the heat pulse due to the absorption of laser energy [8]. Various mechanisms have been proposed to explain AO-HDS, including the spintransfer mechanisms [16-18] and the domain stabilization

\footnotetext{
*weisheng.zhao@buaa.edu.cn

${ }^{\dagger}$ stephane.mangin@univ-lorraine.fr
}

mechanisms $[5,19,20]$. Heat is shown to be important for both types of AOS. However, the correlation between the underlying mechanisms of AO-HDS and AO-HIS still needs to be clarified [21].

Only GdFeCo alloy have shown experimental evidence of both AO-HIS [8,9] and AO-HDS [3]. However, a comprehensive study of the transition between these two types of switching is still lacking. Finding the deterministic parameter(s), which can control the type of switching, would certainly help understand the physical mechanisms and promote practical applications. In this study, we experimentally demonstrate a transition from AO-HIS to AO-HDS in GdFeCo alloy by varying the thickness of the $\mathrm{Pt}$ capping. A similar transition is observed by tuning the laser fluence. We propose that the transition between these two types of AOS might be due to the light absorption profile and the ultrafast heat-transfer process.

\section{METHODS}

Samples with stack of Ta $(3 \quad \mathrm{~nm}) / \mathrm{Pt} \quad(5 \mathrm{~nm}) /$ $\mathrm{Gd}_{x}\left(\mathrm{Fe}_{84} \mathrm{Co}_{16}\right)_{1-x} \quad(20 \mathrm{~nm}) / \mathrm{Pt} \quad(5 \mathrm{~nm}) \quad(x=20.6 \%$ and $20.9 \%$ in atomic percentage) are grown on glass substrates using magnetron sputtering at room temperature. Ta serves as a buffer layer. The thickness of $\mathrm{GdFeCo}$ is consistent with previously reported studies $[3,8]$. The composition of the $\mathrm{GdFeCo}$ is tuned by controlling co-sputtering powers of $\mathrm{Gd}$, $\mathrm{Fe}$, and Co targets mounted in a confocal geometry to allow for alloy deposition [14]. After capping with Pt (5nm), each sample is transferred to another sputtering system equipped with in situ masks. The masks allow depositing different $\mathrm{Pt}$ layers on the same initial sample. We then have Ta $(3 \mathrm{~nm}) / \mathrm{Pt}$ $(5 \mathrm{~nm}) / \mathrm{Gd}_{x}(\mathrm{FeCo})_{1-x}(20 \mathrm{~nm}) / \mathrm{Pt}(\mathrm{Y} \mathrm{nm})(x=20.6 \%$ and $20.9 \%$ ) samples with different $P t$ capping with $Y=5,15$, and $25 \mathrm{~nm}$. The Curie temperature of our samples is estimated to be $550 \mathrm{~K}[22,23]$.

We perform the optical measurements on a setup described elsewhere [14]. The pump laser ( $35 \mathrm{fs}$ pulse duration, $5 \mathrm{kHz}$ repetition rate, $800 \mathrm{~nm}$ wavelength) is incident on the back 


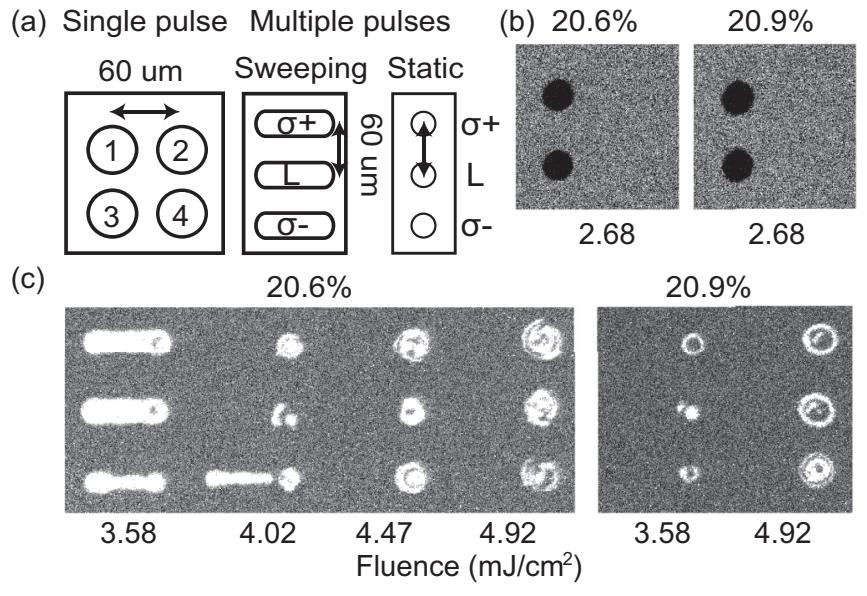

FIG. 1. (a) Schematic illustration of two types of measurements to distinguish between single-pulse AO-HIS and multiple pulses AO-HDS. In the single-pulse measurement, magnetic image is taken after $1,2,3$, or 4 laser pulses. In the multiple-pulse measurement, the films are swept by the beams $\left(\sigma^{+}, \sigma^{-}\right.$, and linear) or are shot by 30000 pulses $\left(\sigma^{+}, \sigma^{-}\right.$, and linear) at the same position. The distance between two spot position shown by the arrows is $60 \mu \mathrm{m}$. (b) MOKE images obtained for the two samples with two compositions and the 5-nm Pt capping layer at $2.68 \mathrm{~mJ} / \mathrm{cm}^{2}$. (c) MOKE images obtained for the 5-nm Pt capping while the laser beam is sweept at $10 \mu \mathrm{m} / \mathrm{s}$ $\left(3.58-4.92 \mathrm{~mJ} / \mathrm{cm}^{2}\right)$ for the three different helicities.

side (the glass side of the sample). The laser fluence is measured with a power meter in the continuous-beam mode before the single-pulse or multiple-pulse experiments. The beam size is determined from the Gaussian fitting of the intensity profile. Figure 1(a) shows the experimental procedure used to distinguish between single-pulse AO-HIS and multiplepulse AO-HDS. To determine the effect of a "single pulse," the measurements by pumping the film with 1,23 and 4 pulses at four different positions are performed. If it results in magnetization switching in one direction for 1 and 3 pulses and in the other direction for 2 and 4 pulses independently of the laser helicity, AO-HIS is concluded. To determine the effect of "multiple pulses," the measurements are performed by either sweeping with the laser beam or pumping with 30000 pulses at the same position (static beam). If it results in a helicity-dependent reversal, AO-HDS is concluded. For samples capped with a 5-nm Pt layer, we imaged with a magneto-optical Kerr (MOKE) microscope on the front side (the capping layer side). For samples capped with thicker Pt layer, the domain imaging is done on the back side to enhance the magnetic contrast.

\section{TRANSITION THICKNESS OF PLATINIUM CAPPING}

Figure 1(b) shows magneto-optic imaging obtained on $\mathrm{Gd}_{x}(\mathrm{FeCo})_{1-x}(20 \mathrm{~nm})$ capped with a $5-\mathrm{nm}$ Pt layer. By pumping with $1,2,3$, and 4 linearly polarized laser pulses of $2.68 \mathrm{~mJ} / \mathrm{cm}^{2}$, typical AO-HIS behavior is demonstrated for the two samples. Indeed, 1 and 3 pulses induce switching of the magnetization orientation while for 2 and 4 pulses, the magnetization remains in its original magnetic orientation.

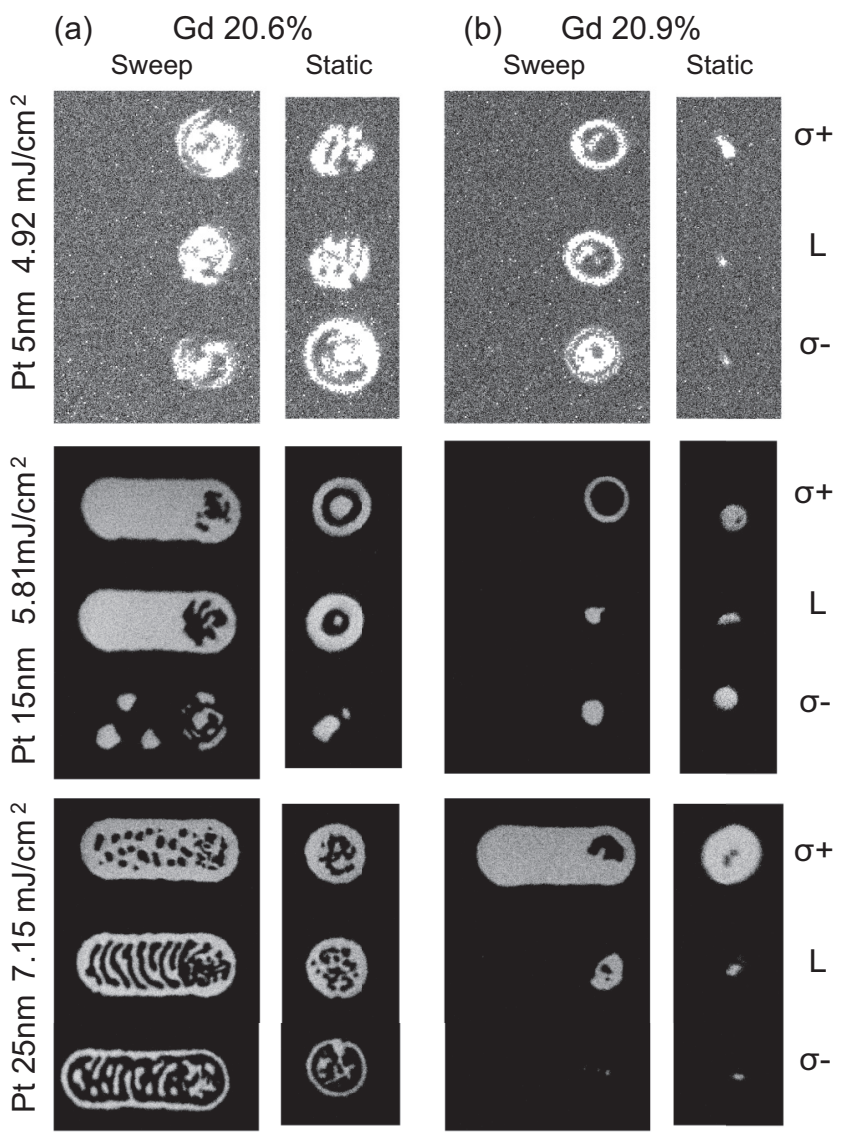

FIG. 2. MOKE images showing the thickness dependence of the AO-HDS effect for the samples with (a) Gd 20.6\% and (b) Gd 20.9\%. The domains are switched by sweeping laser (first column) or static laser (second column). The first, second, and third rows correspond to the results of the sample $\mathrm{Ta} / \mathrm{Pt} / \mathrm{GdFeCo} / \mathrm{Pt}(x \mathrm{~nm})$ with a Pt capping layer of $5 \mathrm{~nm}$ (at $4.92 \mathrm{~mJ} / \mathrm{cm}^{2}$ ), $15 \mathrm{~nm}$ (at $5.81 \mathrm{~mJ} / \mathrm{cm}^{2}$ ), and $25 \mathrm{~nm}$ (at $7.15 \mathrm{~mJ} / \mathrm{cm}^{2}$ ), respectively.

For the case of 5-nm Pt capping layer, AO-HDS is not observed for both Gd compositions (20.6\% and 20.9\%). As shown in Fig. 1(c), the multiple pulses sweeping experiment performed with different helicities shows no helicity dependence. For the sample with $20.6 \% \mathrm{Gd}$ at $3.58 \mathrm{~mJ} / \mathrm{cm}^{2}$ and $4.02 \mathrm{~mJ} / \mathrm{cm}^{2}$ fluence, we observe reversed magnetic domains which are likely created by a domain-dragging effect [24]. When the fluence is above $4.47 \mathrm{~mJ} / \mathrm{cm}^{2}$, we do not observe any switching anymore except at the stop-position of the sweeping path. For the sample with $20.9 \%$ Gd, no switching is observed in the same fluence range.

AO-HDS is only observed on $\mathrm{Gd}_{x}(\mathrm{FeCo})_{1-x}(20 \mathrm{~nm})$ alloys for $x=20.9 \%$ and $20.6 \%$ with thicker Pt capping layer (15 and $25 \mathrm{~nm}$ ). Figure 2 shows the domain patterns induced by the laser beam sweeping and the static 30000 pulses. To quantify the helicity dependence of the phenomena for the two different alloys and the three capping layer thicknesses, the switched ratios for the two helicities are plotted in Fig. 3 starting from a saturated sample.

Figure 3(a) presents the results after the sweeping of $\sigma^{+}$ and $\sigma^{-}$polarized laser beam over the $20.6 \% \mathrm{Gd}$ sample derived from the MOKE images (Fig. 2). For the 15-nm Pt 


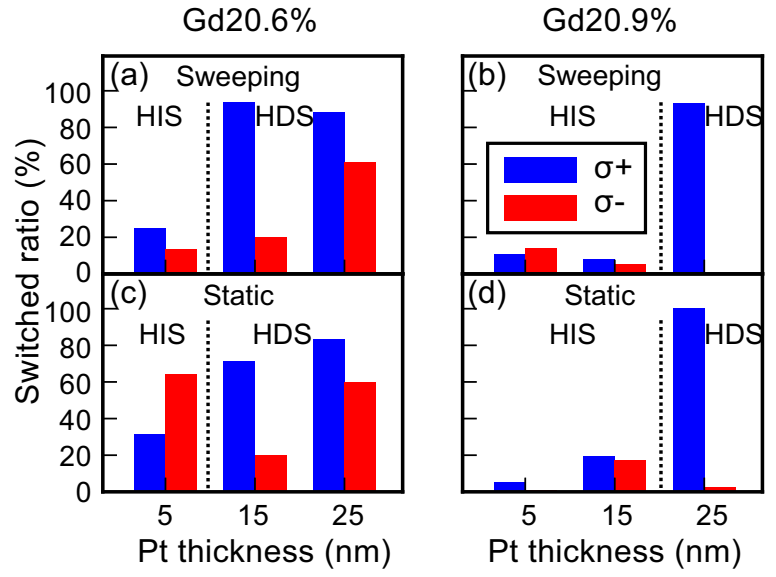

FIG. 3. The switched magnetization ratio induced by $\sigma^{+}$(blue) and $\sigma^{-}$(red) beams as a function of the Pt thickness for the sample of $20.6 \% \mathrm{Gd}$ [(a) and (c)] and $20.9 \% \mathrm{Gd}$ [(b) and (d)]. The pump fluences are $4.92,5.81$, and $7.15 \mathrm{~mJ} / \mathrm{cm}^{2}$ for 5-, 15-, and 25-nm Pt capping, respectively. The raw MOKE images are shown in Fig. 2. The sample was initially saturated.

capping, the switched ratio for $\sigma^{+}$and $\sigma^{-}$are $94 \%$ and $20 \%$, respectively. This switching is clearly helicity-dependent. For the 25-nm Pt capping, the helicity dependency (88\% for $\sigma^{+}$ and $61 \%$ for $\sigma^{-}$) is still observed even though the magnetization could not be fully reversed by circularly polarized beams. The switched ratios induced by static 30000 pulses [Fig. 3(c)] are consistent with the previous results after beam sweeping.

The results for the Gd 20.9\% samples are shown in Figs. 3(b) and 3(d). For 5-nm and 15-nm Pt capping, no AO-HDS can be observed after laser sweeping [Fig. 3(b)]. For the 25-nm-Pt capping, the $\sigma^{+}$helicity beam switches the magnetization $(93 \%)$, while the $\sigma^{-}$helicity beam sets the magnetization orientation to its original direction $(0.2 \%)$. Again, the switching ratios induced by the static 30000 pulses are consistent with the results after laser beam sweeping [Fig. 3(d)]. According to the MOKE image results (Fig. 1) and the switched ratio results (Fig. 3), we can conclude that by increasing the capping Pt layer thickness from $5 \mathrm{~nm}$ to $25 \mathrm{~nm}$, a transition from single-pulse AO-HIS and multiple pulse AO-HDS is demonstrated.

\section{TRANSITION FLUENCE}

For the sample (20.9\% Gd) showing AO-HDS, the switching behavior is further studied as a function of the laser fluence [Fig. 4(a)]. Typical single-pulse AO-HIS is observed at $4.02 \mathrm{~mJ} / \mathrm{cm}^{2}$. At higher fluences (above $4.47 \mathrm{~mJ} / \mathrm{cm}^{2}$ ), multidomain states emerge at the domain center and the single-pulse switching occurs in a ring-shaped region [14]. Figures 4(b) and 4(c) display the MOKE images of multiplepulses AO-HDS induced by a sweeping beam and static 30000 pulses. Remarkably, AO-HDS can also appears in such a sample with higher laser fluences. According to these results, the threshold value of laser fluence for the transition from AO-HIS to AO-HDS is around $5.81 \mathrm{~mJ} / \mathrm{cm}^{2}$.

Figure 4(d) shows the switching ratio induced by single pulses as a function of the laser fluence. The signa-
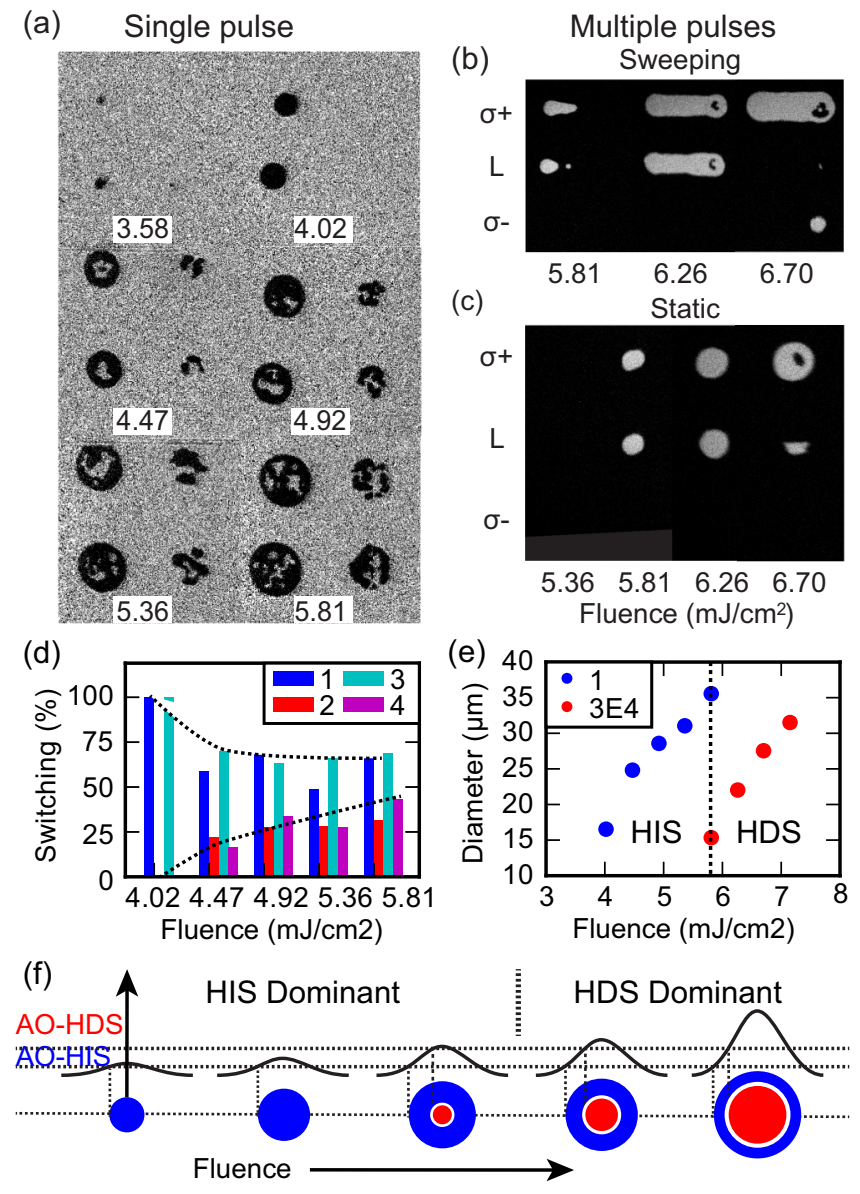

FIG. 4. MOKE images showing the fluence dependence of AOHIS and AO-HDS for the sample Ta/Pt/GdFeCo/Pt $(25 \mathrm{~nm})$ with $\mathrm{Gd}$ $20.9 \%$. Magnetic domains are created by (a) single linear pulses, (b) continuous laser beam of three different helicities in sweeping, and (c) static 30000 pulses of three different helicities. (d) Magnetization switching ratio induced by one (blue), two (red), three (cyan), and four (magenta) pulses. (e) The diameters of switched domains induced by single pulses (red) and 30000 pulses (blue) are shown as a function of the fluence. (f) The laser beam consists of an outer ring $(\mathrm{AO}=\mathrm{HIS}$, blue $)$, an inner core (AO-HDS, red), and an intermediate region (white).

ture of single-pulse switching can be identified from 4.02 to $5.81 \mathrm{~mJ} / \mathrm{cm}^{2}$. A pure AO-HIS effect is observed for $4.02 \mathrm{~mJ} / \mathrm{cm}^{2}$ (100\% switching for 1 and 3 pulses and $0 \%$ for 2 and 4 pulses). However, the switching ratios tend toward 50\% when the laser fluence increases. Figure 4(e) shows the domain diameter as a function of the fluence. Interestingly, the threshold fluence for single-pulse switching is $4.02 \mathrm{~mJ} / \mathrm{cm}^{2}$, much lower than that for multiple-pulse switching $\left(5.81 \mathrm{~mJ} / \mathrm{cm}^{2}\right)$.

Based on the experimental observations, we propose a simplified physical picture to explain the interplay of AO-HIS and AO-HDS [Fig. 4(f)]. The laser beam induces an outer ring (AO-HIS, low fluence), an inner ring (AO-HDS, high fluence), and an intermediate region in between. Those sketches well explain previous experimental observations, including (1) the single-pulse switching in the outer-ring area and the multidomain state in the core [Fig. 4(a)]; (2) the switching ratio as a 
function of laser fluence [Fig. 4(d)]; and (3) the diameter of switched domains as a function of laser fluence [Fig. 4(e)].

\section{DISCUSSION}

The above experimental results enable us to draw three conclusions on AOS effects for GdFeCo alloys. (1) When increasing the capping Pt layer thickness from 5 to $25 \mathrm{~nm}$, a transition from AO-HIS to AO-HDS is observed. (2) When increasing the laser fluence on the $20-\mathrm{nm}$ GdFeCo alloy capped with a $25-\mathrm{nm}$ Pt layer, a transition from AO-HIS to AO-HDS is demonstrated. (3) AO-HIS and AO-HDS show different fluence dependencies, and they are not observed in the same set of experimental parameters.

The transition from single-pulse AO-HIS to multiplepulses AO-HDS by increasing the laser fluence has been explained using the sketch shown in Fig. 4(f). However, it is not clear why the transition from AO-HIS to AO-HDS occurs due to the increase of capping Pt thickness. To answer this question, we analyze the ultrafast heat transfer for different $\mathrm{Pt}$ capping in the following,

We first estimate the laser absorption profile using the transfer matrix method, then calculate the associated electron temperature and the phonon temperature as a function of time using the two-temperature model (Appendix A). Figure 5(a) shows the absorption profile for 5-, 15-, and 25-nm Pt capping. The sample with a 5-nm Pt capping shows a higher absorption compared to the other samples due to a lower reflectance. The reflected powers are $60.1 \%, 65.6 \%$, and $67.3 \%$ for Pt thickness of 5, 15, and $25 \mathrm{~nm}$, respectively. Note that the thicker the Pt capping layer, the stronger the variation of the absorption within the depth of the $20 \mathrm{~nm} \mathrm{GdFeCo}$ layer.

Figure 5(b) shows the deduced electron temperature as a function of depth in GdFeCo calculated using the twotemperature model. For a given laser power, a stronger temperature inhomogeneity within the $\mathrm{GdFeCo}$ layer is present for the thicker Pt capping, which is mainly related to the absorption profile within the GdFeCo layer. We will then assume that the AOS transition comes from the change of the temperature homogeneity.

It has been shown that for AO-HIS, the laser fluence should be higher than the threshold fluence and lower than a certain fluence, i.e., $5.81 \mathrm{~mJ} / \mathrm{cm}^{2}$ in Fig. $4(\mathrm{a})$. It is reasonable to suppose that AO-HIS takes place only if electron temperature ranges between two values, i.e., 2000 and $2500 \mathrm{~K}$. In the sample with 5-nm Pt capping, the magnetization of GdFeCo would tend to show AO-HIS, because the homogeneous electron temperature is ranging between 2000 and $2500 \mathrm{~K}$ in the sample depth [red arrows in Fig. 5(b)]. For 15-nm and 25-nm Pt capping, the electron temperature of a certain part of the GdFeCo depth is lower than the threshold temperature [blue arrows in Fig. 5(b)] due to the in-depth temperature inhomogeneity. The temperature criteria of AO-HIS cannot be fulfilled for all the parts of the GdFeCo layer. In this case, the magnetization of $\mathrm{GdFeCo}$ tends to show multipulse AO-HDS. Figure 5(c) shows the influence of Pt capping on the switching type. In the samples of thin Pt capping, the outer ring (AO-HIS) is dominant, and multiple pulses cannot induce a helicity-dependent switching. In the samples with thick Pt
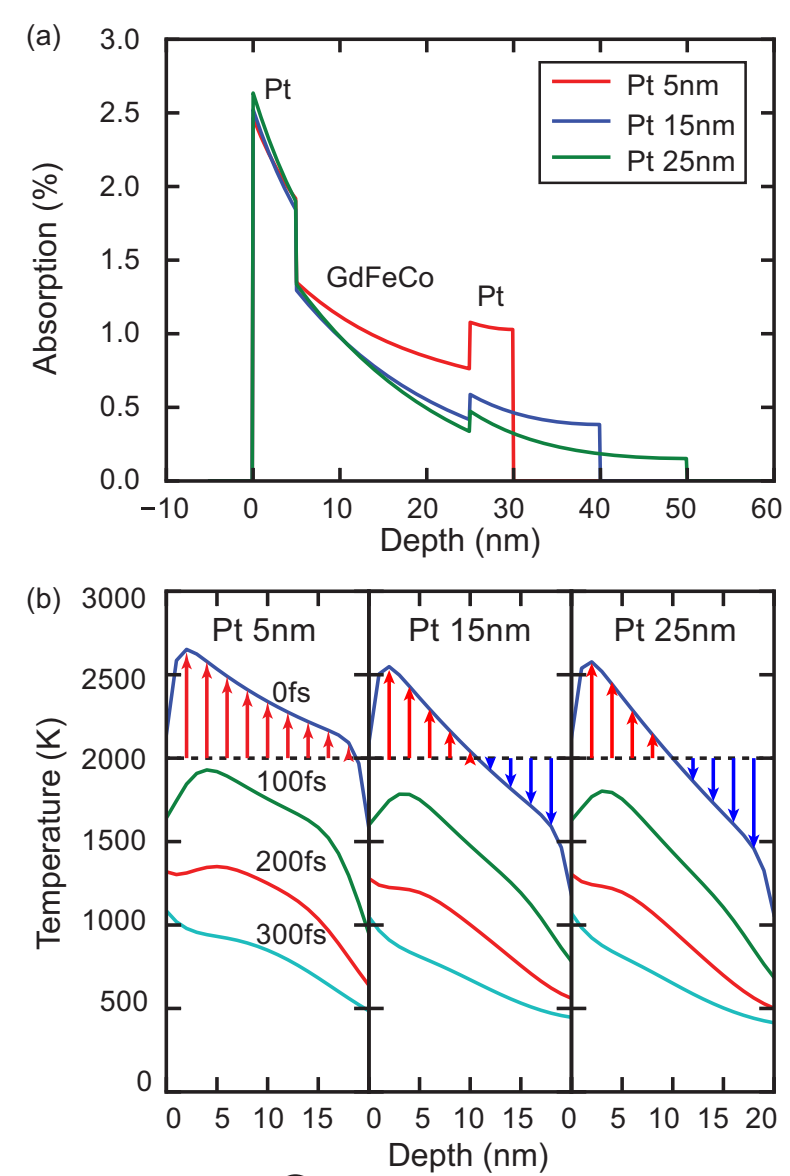

(c)

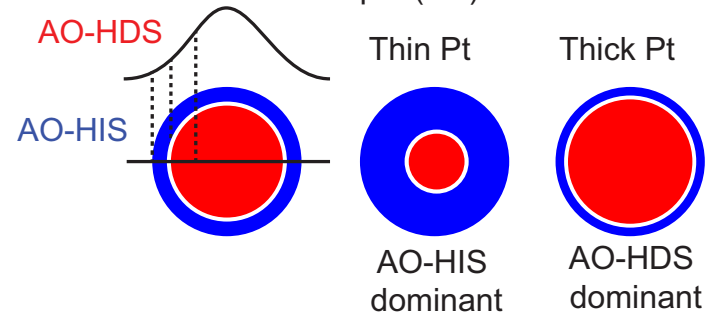

FIG. 5. (a) Calculated light absorption as a function of sample depth for Pt $(5 \mathrm{~nm}) / \mathrm{GdFeCo}(20 \mathrm{~nm}) / \mathrm{Pt}(x \mathrm{~nm}), x=5,15$, and 25 . $\mathrm{Ta}(3)$ is neglected in the calculation. (b) $\mathrm{GdFeCo}$ electron temperature plotted against the depth at 0 (blue), 100 (green), 200 (red), and $300 \mathrm{fs}$ (cyan). $0 \mathrm{fs}$ is defined as the time when the laser pulse stops and the samples are exposed to the laser pulse of $5 \mathrm{~mJ} / \mathrm{cm}^{2}$ during -50 to $0 \mathrm{fs}$. The dashed line indicates the threshold temperature. The magnetization in the hot part (red arrows) tends to switch while the magnetization tends to stay in the original direction in the cold part (blue arrows). (c) The influence of Pt capping: in the case of thin Pt capping, AO-HIS (the outer ring) is dominant; in the case of thick Pt capping, AO-HDS (the inner ring) is dominant.

capping, the inhomogeneous temperature suppresses AO-HIS, and the dominant effect is AO-HDS.

\section{CONCLUSION}

In conclusion, we experimentally demonstrated the transition from single-pulse AO-HIS to multiple-pulse AO-HDS by increasing the Pt capping thickness and by tuning the laser fluence. The two types of switching cannot be observed for 
TABLE I. Parameters for the calculation of the two-temperature model.

\begin{tabular}{lcc}
\hline \hline & $\mathrm{Pt}$ & $\mathrm{GdFeCo}$ \\
\hline$\gamma\left(\mathrm{Jm}^{-3} \mathrm{~K}^{-2}\right)$ & 750 & 167 \\
$C_{p}\left(\mathrm{Jm}^{-1} \mathrm{~K}^{-1}\right)$ & $2.8 \times 10^{6}$ & $2.25 \times 10^{6}$ \\
$\Lambda_{e}\left(\mathrm{Wm}^{-1} \mathrm{~K}^{-1}\right)$ & 50 & 4.5 \\
$\Lambda_{p}\left(\mathrm{Wm}^{-1} \mathrm{~K}^{-1}\right)$ & 7.0 & 2.0 \\
$g_{e p}\left(\mathrm{Wm}^{-1} \mathrm{~K}^{-1}\right)$ & $1.1 \times 10^{18}$ & $0.6 \times 10^{18}$ \\
\hline \hline
\end{tabular}

the same set of parameters, which supports that their mechanisms are uncorrelated. The observation shows that AO-HIS is observed for laser fluencies much lower than AO-HDS in accordance with recent observations of single-pulse switching using electric pulses $[14,15]$. To explain the origin of the transition, the distribution of electron temperature within the $\mathrm{GdFeCo}$ layer on the subpicosecond timescale is calculated based on the two-temperature model. This calculation indicates that the thick Pt capping leads to an inhomogeneous electron temperature within the GdFeCo, which is not compatible with AO-HIS.

\section{ACKNOWLEDGMENTS}

The authors would like to thank Eric Fullerton and Yassine Quessab for fruitful scientific discussion. W.Z. gratefully acknowledge the National Natural Science Foundation of China (Grant No. 61627813), the International Collaboration Project B16001, and the Beijing Advanced Innovation Center for Big Data and Brain Computing (BDBC). Y.X. is thankful for the support from the China Postdoctoral Science Foundation (Grant No. 2017M620569). X.L. acknowledges the support of Young Elite Scientists Sponsorship Program by CAST (Grant No. 2018QNRC001) and the Fundamental Research Funds for the Central Universities of China. This work was supported by the ANR- 15-CE24-0009 UMAMI and by the ANR-Labcom Project LSTNM, by the Institut Carnot ICEEL for the project «Optic-switch » and Matelas and by the French PIA project "Lorraine Université d'Excellence," reference ANR-15-IDEX-04-LUE. Experiments were performed using equipment from the TUBE. Davm funded by FEDER (EU), ANR, Région Grand Est and Metropole Grand Nancy.

\section{APPENDIX A: TWO TEMPERATURE MODEL}

The two-temperature model considers the electron channel and the phonon channel for heat propagation. We calculate the electron temperature by solving the Eq. (A1) numerically. The parameters used in the model are shown in Table I.

$$
\begin{aligned}
C_{e} \frac{\partial T_{e}}{\partial t} & =g_{e p}\left(T_{p}-T_{e}\right)+\Lambda_{e} \frac{\partial^{2} T_{e}}{\partial x^{2}}, \\
C_{p} \frac{\partial T_{p}}{\partial t} & =g_{e p}\left(T_{e}-T_{p}\right)+\Lambda_{p} \frac{\partial^{2} T_{p}}{\partial x^{2}} .
\end{aligned}
$$

The interfacial thermal conductance of the electrons between Pt and GdFeCo is unknown to our knowledge. We use $5 \times 10^{9} \mathrm{Wm}^{-2} \mathrm{~K}^{-1}$ which is reported by Choi et al for the interfacial conductance for $\mathrm{Pt} / \mathrm{Au}$ interface [25].

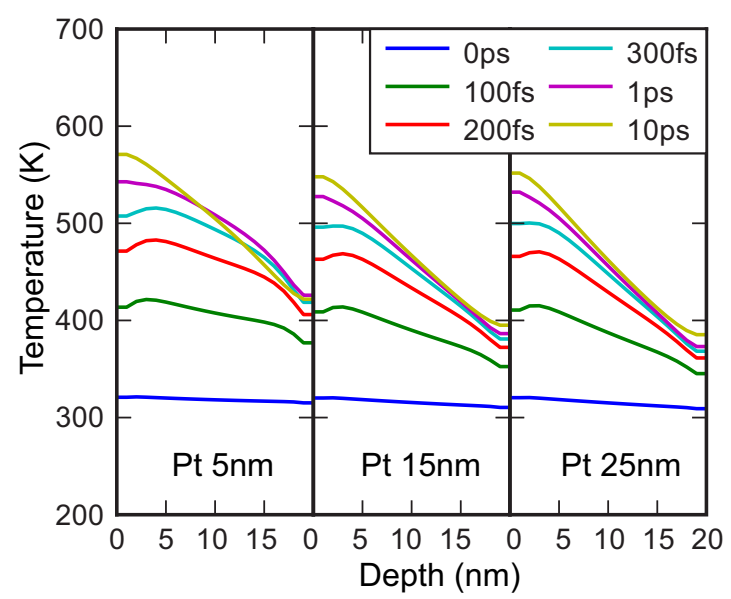

FIG. 6. Calculated GdFeCo phonon temperature plotted against the depth at 0 (blue), 100 (green), 200 (red), and 300 fs (cyan). 0 fs is defined as the time when the laser pulse stops and the samples are exposed to the laser pulse of $5 \mathrm{~mJ} / \mathrm{cm}^{2}$ during -50 to $0 \mathrm{fs}$.

\section{APPENDIX B: PHONON TEMPERATURE AND MCD}

Figure 6 shows the GdFeCo phonon temperature calculated by the two-temperature model. Note that the Curie temperature in our sample is estimated to be $550 \mathrm{~K}$. The phonon temperature at $10 \mathrm{ps}$ reaches $550 \mathrm{k}$, which is close to the Curie temperature.

We demonstrated in Fig. 4 AO-HIS at low fluences and AO-HDS at high fluences. The multiple-pulse AO-HDS could be potentially explained by the MCD in GdFeCo [18]. We performed the standard ellipsometry measurement to evaluate the MCD in our sample. First we measure the refractive index for the $\mathrm{M}+$ and $\mathrm{M}-$ states, and then the refractive index of GdFeCo for linearly polarized (LP) is given as $n_{L P}=\left(n_{M+}+n_{M-}\right) / 2$ and $\Delta n=n_{M+}-n_{M-}$. Then the refractive index of $\mathrm{GdFeCo}$ for left-handed circularly polarized (LC) and right-handed circularly polarized (RC) light are

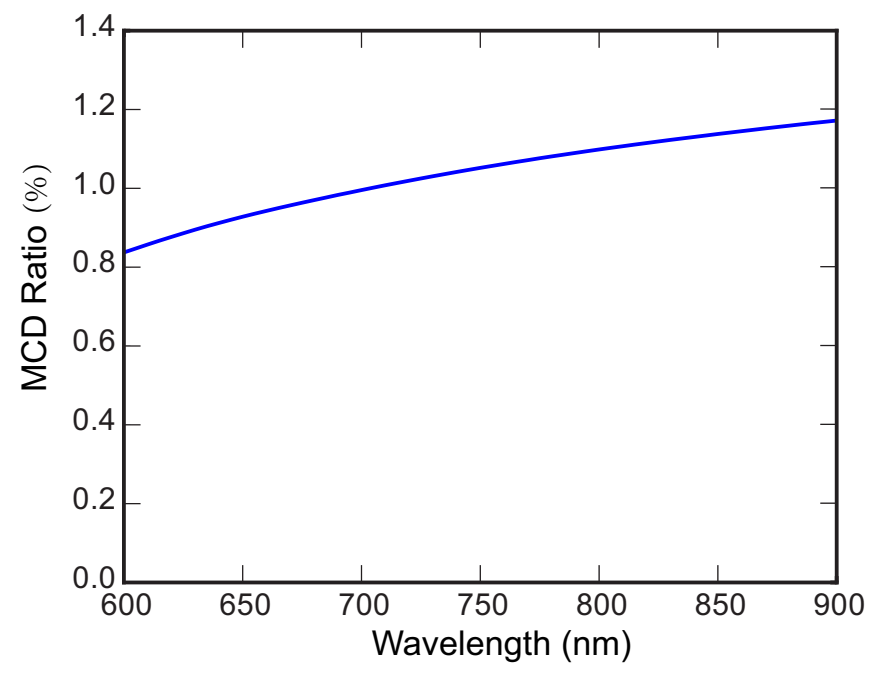

FIG. 7. MCD ratio deduced from ellipsometry measurement is shown as a function of wavelength. 
$n_{\mathrm{LC}}=n_{\mathrm{LP}}+\frac{1}{2} \Delta n$ and $n_{\mathrm{RC}}=n_{\mathrm{LP}}-\frac{1}{2} \Delta n$. Based on the refractive index for LC and RC, the MCD ratio is calculated for the wavelength range 600-900 $\mathrm{nm}$ (Fig. 7). Our sample shows an MCD ratio of $1.1 \%$ at $800 \mathrm{~nm}$.
[1] E. Beaurepaire, J.-C. Merle, A. Daunois, and J.-Y. Bigot, Ultrafast Spin Dynamics in Ferromagnetic Nickel, Phys. Rev. Lett. 76, 4250 (1996).

[2] A. Kirilyuk, A. V. Kimel, and T. Rasing, Controlling spins with light, Philos. Trans. R. Soc. Lond. Math. Phys. Eng. Sci. 369, 3631 (2011).

[3] C. D. Stanciu, F. Hansteen, A. V. Kimel, A. Kirilyuk, A. Tsukamoto, A. Itoh, and Th. Rasing, All-Optical Magnetic Recording with Circularly Polarized Light, Phys. Rev. Lett. 99, 047601 (2007).

[4] K. Vahaplar, A. M. Kalashnikova, A. V. Kimel, D. Hinzke, U. Nowak, R. Chantrell, A. Tsukamoto, A. Itoh, A. Kirilyuk, and Th. Rasing, Ultrafast Path for Optical Magnetization Reversal via a Strongly Nonequilibrium State, Phys. Rev. Lett. 103, 117201 (2009).

[5] S. Mangin, M. Gottwald, C.-H. Lambert, D. Steil, V. Uhlîr, L. Pang, M. Hehn, S. Alebrand, M. Cinchetti, G. Malinowski, Y. Fainman, M. Aeschlimann, and E. E. Fullerton, Engineered materials for all-optical helicity-dependent magnetic switching, Nat. Mater. 13, 286 (2014).

[6] C.-H. Lambert, S. s. Mangin, B. s. d. c. s. Varaprasad, Y. k. Takahashi, M. Hehn, M. Cinchetti, G. Malinowski, K. Hono, Y. Fainman, M. Aeschlimann, and E. e. Fullerton, All-optical control of ferromagnetic thin films and nanostructures, Science 345, 1337 (2014).

[7] M. S. El Hadri, P. Pirro, C.-H. Lambert, S. Petit-Watelot, Y. Quessab, M. Hehn, F. Montaigne, G. Malinowski, and S. Mangin, Two types of all-optical magnetization switching mechanisms using femtosecond laser pulses, Phys. Rev. B 94, 064412 (2016).

[8] T. A. Ostler et al., Ultrafast heating as a sufficient stimulus for magnetization reversal in a ferrimagnet, Nat. Commun. 3, 666 (2012).

[9] I. Radu, K. Vahaplar, C. Stamm, T. Kachel, N. Pontius, H. A. Dürr, T. A. Ostler, J. Barker, R. F. L. Evans, R. W. Chantrell, A. Tsukamoto, A. Itoh, A. Kirilyuk, T. Rasing, and A. V. Kimel, Transient ferromagnetic-like state mediating ultrafast reversal of antiferromagnetically coupled spins, Nature 472, 205 (2011).

[10] M. L. M. Lalieu, M. J. G. Peeters, S. R. R. Haenen, R. Lavrijsen, and B. Koopmans, Deterministic all-optical switching of synthetic ferrimagnets using single femtosecond laser pulses, Phys. Rev. B 96, 220411(R) (2017).

[11] J.-Y. Chen, L. He, J.-P. Wang, and M. Li, All-Optical Switching of Magnetic Tunnel Junctions with Single Subpicosecond Laser Pulses, Phys. Rev. Appl. 7, 021001 (2017).

[12] M. S. E. Hadri, P. Pirro, C.-H. Lambert, N. Bergeard, S. Petit-Watelot, M. Hehn, G. Malinowski, F. Montaigne, Y. Quessab, R. Medapalli, E. E. Fullerton, and S. Mangin, Electri- cal characterization of all-optical helicity-dependent switching in ferromagnetic Hall crosses, Appl. Phys. Lett. 108, 092405 (2016).

[13] R. B. Wilson, Ultrafast magnetic switching of GdFeCo with electronic heat currents, Phys. Rev. B 95, 180409(R) (2017).

[14] Y. Xu, M. Deb, G. Malinowski, M. Hehn, W. Zhao, and S. Mangin, Ultrafast magnetization manipulation using single femtosecond light and hot-electron pulses, Adv. Mater. 29, 1703474 (2017).

[15] Y. Yang, R. B. Wilson, J. Gorchon, C.-H. Lambert, S. Salahuddin, and J. Bokor, Ultrafast magnetization reversal by picosecond electrical pulses, Sci. Adv. 3, e1603117 (2017).

[16] M. Berritta, R. Mondal, K. Carva, and P. M. Oppeneer, Ab Initio Theory of Coherent Laser-Induced Magnetization in Metals, Phys. Rev. Lett. 117, 137203 (2016).

[17] G.-M. Choi, A. Schleife, and D. G. Cahill, Optical-helicitydriven magnetization dynamics in metallic ferromagnets, Nat. Commun. 8, 15085 (2017).

[18] J. Gorchon, Y. Yang, and J. Bokor, Model for multi-shot allthermal all-optical switching in ferromagnets, Phys. Rev. B 94, 020409(R) (2016).

[19] M. S. El Hadri, M. Hehn, P. Pirro, C.-H. Lambert, G. Malinowski, E. E. Fullerton, and S. Mangin, Domain size criterion for the observation of all-optical helicity-dependent switching in magnetic thin films, Phys. Rev. B 94, 064419 (2016).

[20] A. Hassdenteufel, J. Schmidt, C. Schubert, B. Hebler, M. Helm, M. Albrecht, and R. Bratschitsch, Low-remanence criterion for helicity-dependent all-optical magnetic switching in ferrimagnets, Phys. Rev. B 91, 104431 (2015).

[21] A. R. Khorsand, M. Savoini, A. Kirilyuk, A. V. Kimel, A. Tsukamoto, A. Itoh, and Th. Rasing, Role of Magnetic Circular Dichroism in All-Optical Magnetic Recording, Phys. Rev. Lett. 108, 127205 (2012).

[22] C. D. Stanciu, A. V. Kimel, F. Hansteen, A. Tsukamoto, A. Itoh, A. Kirilyuk, and Th. Rasing, Ultrafast spin dynamics across compensation points in ferrimagnetic GdFeCo: The role of angular momentum compensation, Phys. Rev. B 73, 220402(R) (2006).

[23] T. A. Ostler, Crystallographically amorphous ferrimagnetic alloys: Comparing a localized atomistic spin model with experiments, Phys. Rev. B 84, 024407 (2011).

[24] Y. Quessab, R. Medapalli, M. S. El Hadri, M. Hehn, G. Malinowski, E. E. Fullerton, and S. Mangin, Helicity-dependent all-optical domain wall motion in ferromagnetic thin films, Phys. Rev. B 97, 054419 (2018).

[25] G.-M. Choi, R. B. Wilson, and D. G. Cahill, Indirect heating of $\mathrm{Pt}$ by short-pulse laser irradiation of $\mathrm{Au}$ in a nanoscale $\mathrm{Pt} / \mathrm{Au}$ bilayer, Phys. Rev. B 89, 064307 (2014). 